Cultures \& Conflits

03 | automne 1991

Mafia, drogue et politique

\title{
Les réseaux de la drogue dans le triangle d'or
}

\author{
Martial Dassé
}

\section{OpenEdition}

Journals

Édition électronique

URL : http://journals.openedition.org/conflits/111

DOI : $10.4000 /$ conflits. 111

ISSN : $1777-5345$

Éditeur :

CCLS - Centre d'études sur les conflits lilberté et sécurité, L'Harmattan

Édition imprimée

Date de publication : 17 octobre 1991

ISSN : 1157-996X

\section{Référence électronique}

Martial Dassé, "Les réseaux de la drogue dans le triangle d'or », Cultures \& Conflits [En ligne], 03 |

automne 1991, mis en ligne le 31 décembre 2002, consulté le 30 mars 2021. URL : http://

journals.openedition.org/conflits/111; DOI : https://doi.org/10.4000/conflits.111

Ce document a été généré automatiquement le 30 mars 2021.

Creative Commons License 


\title{
Les réseaux de la drogue dans le triangle d'or
}

\author{
Martial Dassé
}

Le problème de la drogue en Asie du Sud-Est ne se limite plus à la Birmanie, la Thaillande et le Laos, pays composant le Triangle d'or. Certes, ces pays sont les principaux producteurs et raffineurs de l'opium cultivé par leurs minorités montagnardes. Mais le rôle de pays de transit joué par la Malaisie, Singapour et les Philippines n'est pas moins important et criminel. Seuls, le Cambodge et Brunéi ne sont pas concernés. Quant à l'Indonésie elle lutte sévèrement contre tous les réseaux de banditisme par les mêmes méthodes que celles utilisées contre les communistes : les exécutions sommaires.

Dans chacun des pays, les trafiquants appartiennent à des catégories différentes. En Birmanie, ce sont de véritables guérillas ethniques ou des groupes de bandits, plutôt que le gouvernement, qui contrôlent les paysans montagnards, leur achètent l'opium, le raffinent en héroïne et le vendent ensuite aux intermédiaires qui se chargent de l'expédition. Ces guérillas se justifient en affirmant que c'est l'un des rares moyens de financer leur "mouvement de libération" contre le gouvernement de Rangoun. Ainsi, l'opium est érigé au rang de nerf de la guerre. Au Laos, le gouvernement n'agit pas, sous prétexte qu'en permettant la culture du pavot il pacifie 400 à 500000 montagnards Méo, toujours en révolte larvée, et soutenus par les Etats-Unis, la Chine ou la Thaïlande selon les moments. Seulement cet argument de la "légitime défense" évoqué par le gouvernement ne résiste pas à un examen sérieux. La Malaisie, Singapour, les Philippines et la Thaillande apparaissent quant à eux très nettement comme des pays trafiquants. En Malaisie et à Singapour, les autorités ont pour politique d'interdire l'entrée de la drogue, mais d'en permettre l'exportation. Aux Philippines, pays où tout va à vau-l'eau, le gouvernement est dépassé car ce sont les organismes de répression qui pratiquent le trafic de toutes les drogues possibles. Au Vietnam, c'est surtout la marijuana du Laos qui part vers les États-Unis. Ainsi, les autorités ne veulent pas ou ne peuvent pas mettre fin au trafic car se serait confronter le pouvoir policier, à celui des militaires ou à celui des partis politiques. La paix intérieure vaut bien alors de fermer 
les yeux sur une drogue qui ne fait que passer. Aux Occidentaux de trouver les solutions.

La tendance actuelle est en effet de considérer de plus en plus la drogue comme une marchandise comme les autres, dépendant de l'offre et de la demande. Les 2400 tonnes d'opium de Birmanie, les 350-400 tonnes du Laos et les 40-60 tonnes de Thaillande se réduisent à environ 2000 tonnes après que les consommateurs locaux aient été pourvus. Cela signifie 800 tonnes d'héroïne qui sont vendues là où se trouvent des acheteurs. Nul n'échappe plus au fléau, le dernier pays touché étant la Chine ellemême. La drogue rapporte trop d'argent pour que ses victimes soient prises en considération. D'un seul élan, toute l'Asie pense que nul n'est obligé de consommer de la drogue, qu'il s'agit donc d'un problème personnel et non étatique. Seule, la Chine, qui craint une résurgence de l'opiomanie prend des mesures de lutte extrêmement strictes.

I.- Production et légitimation du trafic

1.1.- La Birmanie : les enjeux géopolitiques

La guerre du KMT contre la Chine

L'histoire du développement de la culture du pavot et de son complément, le trafic d'héroïne, a été déjà largement traitée et analysée(1). Après la victoire de Mao en 1949, des débris du Kuomintang (KMT) se réfugient dans le nord de la Birmanie. Là, aidés par les Etats-Unis qui, naïvement, croient possible une reconquête de la Chine, ils se reconstituent et se nomment pompeusement troisième et cinquième armées. Jamais ces vaincus n'eurent l'illusion de pouvoir battre l'armée populaire, ni ne crurent que le peuple se soulèverait à leur appel, mais, en faisant semblant de croire à cette croisade anti-communiste, ils pouvaient survivre et mettre sur pied le trafic de drogue, infiniment moins dangereux et plus rentable, en prétendant en outre que c'était là un moyen de financer le combat pour la démocratie.

Seulement battus par l'armée populaire lorsqu'ils rentrent en Chine, et ensuite par l'armée birmane, le KMT quitte la Birmanie et se replie en Thaïlande en 1953 et 1954. Les responsables des troisième et cinquième armées y sont reçus par leur complice local, le général Pao Sryanond, directeur général de la police thaïlandaise, et homme fort de la CIA chargé de lutter contre le communisme. Sous cette protection, la drogue part sans encombre vers l'Occident. En 1957, le général Pao rate un coup d'État et s'exile en Suisse où il mourra. Mais les militaires thaillandais s'emparent du trafic, qu'ils poursuivent, de manière plus discrète, toujours avec le KMT, sous couvert de lutte anticommuniste et protégé par la CIA.

Le KMT et les minorités

En 1960, pratiquement toutes les minorités de Birmanie(2) sont en révolte contre le gouvernement de Rangoun. Le KMT soutient l'Armée pour l'indépendance Kachin et s'associe avec les frères Lo Hsing-han et Lo Hsing-minh, chinois du district de Kokang(3). Les frères Lo, pour donner une couverture à leur trafic montent avec la cinquième armée, un pseudo-mouvement de libération nommé l'Armée révolutionnaire de l'État Shan (ARES). Mais, en même temps, les frères Lo bénéfcient aussi de la protection du gouvernement birman car ils ont constitué une milice anti-communiste (Kha Kwe Yei). Ainsi, les frères Lo et le KMT jouissent-ils simultanément de la protection de la Birmanie puisqu'ils luttent contre les maquis communistes, de celle de la CIA afin qu'ils envoient des agents en Chine, et de celle de la Thaïlande qui pense que ces conflits browniens affaiblissent utilement ces ennemis héréditaires que sont la 
Birmanie et la Chine. Le trafic de drogue est alors péché moins que véniel eu égard aux enjeux géopolitiques.

Seulement en 1973, la Birmanie, s'apercevant que les chefs des milices deviennent dangereux car ils se transforment en seigneurs de la guerre incontrôlables, donne l'ordre de dissolution des milices. Les frères Lo refusent. En juillet de la même année, l'extrême-droite thaïlandaise fait arrêter Lo Hsing-han à Mae Hongson (ville thaïlandaise du Nord-Ouest à quelques kilomètres de la frontière birmane) par la police des frontières. Il est immédiatement remis à la Birmanie qui le condamne à mort puis commue la sentence à la prison à perpétuité. En effet, si Lo et le KMT collaborent avec les militaires thaïlandais, l'extrême-droite traditionaliste a elle un autre poulain à pousser, Khun Sa.

Khun Sa contre le KMT

Khun Sa' a débuté en 1950 dans les rangs de la troisième armée du KMT. En 1965, il vole de ses propres ailes et constitue une Kha Kwe Yei. Grand organisateur, fin stratège et sans scrupule, il émerge rapidement comme un seigneur de la drogue. S'opposant au KMT, il s'allie avec le commandant en chef de l'armée laotienne le général Ouan Rattikone qui est déjà lié à l'extrême-droite traditionaliste thaïlandaise. En 1969, devenu trop gênant, il est arrêté par l'armée birmane. Au début de 1973, il est libéré contre deux médecins coopérants soviétiques enlevés par l'Armée de l'État Shan (AES) véritablement composée en majorité de Shan, mais qui se consacre surtout au banditisme. L'arrestation de Lo Hsing-han arrive à point nommé. En outre, en octobre 1973, une révolution étudiante met fin au régime militaire des maréchaux thaïlandais5 conférant le pouvoir aux traditionalistes de toutes les tendances.

Dès lors, ils replacent Khun Sa et s'en servent dans leur lutte contre les militaires qui sont maintenant sur la défensive. Khun Sa établit deux bases à Mae Hongson, sous l'égide de son oncle Khun Seng, et dans la province de Chieng Maï. Avec l'aide de AES, il constitue l'Armée unifiée Shan (AUS). Durant la dictature de l'extrême-droite traditionaliste(6), de nombreux trafiquants sont arrêtés et quelques uns fusillés, mais ce sont toujours ceux ayant des relations avec le KMT. Par contre, Khun Sa étale ses liens avec l'extrême-droite(7). Sous prétexte d'aider à la construction de routes dans les zones infiltrées par les communistes thaïlandais, Khun Sa rétablit des bases pour l'AUS. Après le retour des militaires sur la scène politique, en septembre 1978 le général Kriangsak tente encore d'aider le KMT en leur rendant visite pour rendre hommage à leur rôle dans la lutte contre le communisme. Mais il est déjà trop tard pour eux. Khun Sa est trop puissant.

En juin 1980, Lo Hsing-han est amnistié. En échange, il accepte de se remettre au service de Rangoun. Il ouvre un camp à Lashio (État Shan) pour l'entraînement de milices de montagnards. Son frère Lo Hsing-minh abandonne l'ARES et la cinquième armée en août suivant pour le rejoindre. Cela affaiblit d'autant le KMT qui est harcelé par Khun Sa. Enfin, le 11 mars 1984, Khun Sa fait sauter, à l'aide de 7000 bâtons de dynamite, à Chieng Maï, le quartier général de la troisième armée, qui se rallie. L'Armée révolutionnaire unifiée Shan qui servait de paravent à la troisième armée devient l'Armée taï unifiée de libération(7) sous la tutelle de Khun Sa. Du KMT il ne reste que quelques centaines d'hommes de la cinquième armée. Seulement, si Khun Sa a détruit le KMT, il lui faut encore dominer les frères Lo et Peung pour être en position de "monopoleur"

Khun Sa contre les frères Peung 
En 1966, la Chine avait décidé d'aider le Parti communiste de Birmanie (PCB) qui subissait défaite sur défaite dans la plaine centrale. L'armée populaire était alors intervenue directement pour créer des bases arrières dans le district de Kokang. Pour obtenir le soutien de la population anti-communiste, la Chine s'adressait à l'époque aux frères Peung Kya-shin et Peung Kya-fu, anciens comparses des frères Lo. Ils acceptaient de collaborer avec le PCB à condition d'avoir les mains libres pour reprendre le trafic. A partir de ce moment, les frères Peung renforcent leurs positions en utilisant toujours comme paravent un vocabulaire révolutionnaire, et en créant une Force révolutionnaire de l'État du Kokang (FREK), qui constitue une des premières "guérillas communistes" du PCB. La FREK dispose d'une infrastructure mais pas encore d'assez d'opium. Ce n'est qu'en 1973, que le PCB, soutenu par l'armée populaire, peut s'emparer des États Wa, situés en dessous du Kokang, le long de la frontière chinoise. Les montagnards Wa se soumettent mais à la condition de pouvoir intensifier la culture du pavot. Ils s'érigent en douzième brigade Wa qui représentera la principale force du PCB avec 6 à 8000 hommes. La jonction est faite avec la FREK Le PCB crée un Département de l'opium, géré par la FREK, qui raffine l'opium en héroïne et la vend à Khun Sa. Cependant, la guérilla ne progresse pas et coûte extrêmement cher en hommes aussi bien à la FREK qu'à la douzième brigade. Les militaires birmans profitent de ce mécontentement et envoient les frères Lo proposer un accord aux frères Peung: s'ils se révoltent contre le PCB en entraînant les Wa avec eux, les militaires leur accorderont toute facilité pour leur trafic. Les frères Peung acceptent. Le 14 mars 1989, la FREK se soulève contre le $\mathrm{PCB}$, se transformant en Armée nationale démocratique du Kokang (ANDK). Le 12 avril, la douzième brigade Wa s'insurge à son tour et devient l'Armée des États unifiés Wa (AEUW). En mai c'en est fait du PCB dont les membres du Bureau politique se réfugient en Chine. La guerre de la drogue entre dans une nouvelle étape. Les frères Peung rompent leur alliance avec Khun Sa, se lient à nouveau avec les frères Lo, et s'associent aussi avec l'AEUW Le quasi-monopole de Khun Sa s'écroule. La guérilla entre l'ANDK et l'AEUW d'un côté, Khun Sa et l'AES de l'autre fait des centaines de victimes et prend une nouvelle intensité . Les militaires birmans se frottent les mains: la "paix" est revenue dans l'État Shan où ils n'ont plus à se battre mais seulement à compter les points. Les Chinois Lo, Pung et I ?iun Sa s'exterminent par montagnards interposés afin de s'assurer le contrôle de l'opium.

1.2.- Le Laos : la pacification des Méo

Lors de la deuxième guerre d'Indochine, les militaires laotiens se révélant peu fiables, les Américains organisèrent une "armée secrète" de Méo sous la direction de l'un des leurs, le général Vang Pao. Trop occupé par la lutte contre les troupes vietnamiennes, ils n'avaient plus de temps à consacrer à la culture du pavot et, en ce cas précis, il est faux de dire que les Américains facilitèrent cette culture pour obtenir leur appui. Au contraire, la production d'opium, de 25 à 50 tonnes selon les années, était à peine supérieure aux besoins. Le surplus était pris en main par le général Ouan Rattikone.

Après la prise du pouvoir en 1975 par le parti révolutionnaire du peuple Lao la production atteint de manière régulière les 40-50 tonnes. Toutefois, elle augmente lentement du fait que, des États-Unis où il s'est réfugié, le général Vang Pao, avec le soutien de la CIA, de la Thaïlande et de la Chine, continue à diriger une guérilla anticommuniste. Pour "acheter" les Méo, dès 1978, le gouvernement laotien met sur pied un plan de développement sous la tutelle du ministère des Finances. Ce dernier est responsable à la fois de l'achat aux montagnards, du raffinement et de l'exportation. Pour un pays aussi pauvre, c'est une aubaine. La culture du pavot ne demande aucun 
soin et son raffinement ne coûte presque rien. Dès lors la production progresse d'autant plus que les Méo préfèrent nettement le travail du paysan qui procure un revenu sûr et facile, aux dangers de la guérilla. En 1984, la production est de 50-75 tonnes, en 1987 de 200-250 tonnes et en 1990 de 350-400 tonnes. Le pavot devient la principale ressource agricole du Laos et non plus seulement un moyen de pacifier les montagnards. Bien que critiquant régulièrement le Laos, les Etats-Unis ne veulent en aucun cas gêner un pays qui prétend tout faire pour retrouver les dépouilles des militaires américains disparus au cours de la guerre. De peur de mettre fin à cette "bonne volonté" Washington laisse ses citoyens s'empoisonner avec l'héroïne en provenance du Laos. Le Laos peut, en toute impunité et sécurité, poursuivre son trafic, Washington se contentant de plates protestations. Le contraste est frappant avec l'attitude de Washington à l'égard de la Birmanie, qui n'a pas de corps de soldats comme monnaie d'échange, et est confrontée à toute la hargne et la frustration des diplomates et représentants du Congrès américain.

II.- Commercialisation et extension transnationale du trafic

2.1.-La Thaillande

La Thaïlande est le plus important pays de transit. Elle réceptionne l'héroïne de Birmanie et du Laos, et en fabrique aussi elle-même dans des laboratoires situés sur la frontière birmane. L'héroïne est expédiée vers les Etats-Unis à partir de Bangkok. Une deuxième partie part vers la Malaisie et l'Indonésie, et une troisième vers les Philippines. La proportion de ce partage de la distribution est inconnue.

La Thaillande prévoit, depuis 1979, la peine de mort pour toute personne en possession de $100 \mathrm{~g}$ d'héroïne, mais jamais cette peine n'a été appliquée à des Occidentaux. La Thaïlande cherche trop en effet à s'enrichir pour prendre un air moralisateur en exécutant des boucs émissaires. Ce serait une véritable provocation devant sa profonde et évidente implication dans le trafic. De plus, les trafiquants arrêtés sont surtout des Nigérians à l'aéroport de Bangkok. Aujourd'hui, le conflit CIA-KMT-militaires thaïlandais contre Khun Sa-extrême-droite traditionaliste, pour savoir qui contrôlerait les minorités et le "magot", est en voie de disparition. Après l'échec des tentatives de monopolisation, il y a une "dispersion" du trafic, un retour à une "saine" concurrence. Les Chinois qui assurent la commercialisation paient tous ceux dont ils ont besoin sans regarder l'étiquette politique. Les policiers ne s'intéressent, comme aux Philippines, qu'à augmenter leurs revenus sans se préoccuper des incidences internationales. Ils protègent tous ceux qui en ont les moyens, que ce soit Khun Sa, le KMT, les frères Lo et Peung ou les représentants du gouvernement laotien. Comme pour le conflit du Cambodge, la Thaïlande est neutre, c'est-à-dire profite tous azimuts du fait qu'on a besoin de son territoire. Elle se conduit en commerçant pour qui "un client est un client"

\subsection{La Malaisie, Singapour et le Vietnam}

Chaque année, les Etats-Unis dénoncent la Malaisie comme un centre de raffinage et une plaque tournante de l'exportation la drogue vers l'Australie et l'Europe. Seulement ces condamnations ne sont suivies d'aucune action. Dès lors la Malaisie peut se donner l'image d'un pays "pur et dur" dans son combat contre les trafiquants. Ne prévoit-elle pas la ne de mort envers toute personne en possession de $15 \mathrm{~g}$. héroïne, depuis 1975 ? En 1983, n'a-t-elle pas imposé cette peine mort comme obligatoire non seulement pour 15 g. d'héroïne mais aussi pour 200 g. de marijuana? Depuis 1983 encore, n'a-t-il pas condamné à mort 385 personnes dont 126 ont été exécutées parmi elles deux 
Australiens et un Britannique. Faut-il ajouter e 786 personnes sont toujours en attente de jugement? Le mai 1990, le gouvernement malais n'a-t-il pas pendu d'un coup 7 Chinois et une Chinoise de HongKong, qui avaient été arrêtés en 1982, porteurs de 12,7 $\mathrm{kg}$ d'héroïne?

En réalité, cette dureté tape-à-l'oeil n'est qu'hypocrisie. Les Occidentaux exécutés ne sont que de lamentables drogués venus se ravitailler sur place à peu de frais; en revanche, la Malaisie, par bateaux en provenance du sud de la Thaillande et de la Birmanie, reçoit des dizaines de tonnes d'héroïne et pourtant aucun de ces vrais trafiquants n'a jamais été arrêté et encore moins pendu. De plus c'est de Malaisie que partent vers la Thaillande tous les produits chimiques nécessaires à la transformation de l'opium en héroïne, et en particulier l'acide anhydride. Seulement, là encore, rien n'est fait car cela remettrait en cause trop de relations dans la zone.

A Singapour, on n'est pas en reste. La loi de 1975, identique à celle de la Malaisie, a été perfectionnée en février 1990. La peine de mort est maintenant prévue pour la possession de $500 \mathrm{~g}$. de marijuana, $30 \mathrm{~g}$. de cocaïne et 1,2 kg d'opium. Depuis 1975, 26 personnes ont été exécutées et 37 sont en instance de l'être. Certes et contrairement à la Malaisie, la police ne demeure pas totalement inactive; en 1978, elle a même démantelé un vaste réseau, qualifié de "Chinese Connection". Pourtant Singapour reste un centre important de transit et de blanchiment de l'argent ; or, dans ce pays de 2,6 millions d'habitants, isolés sur une île, où règne l'ordre et où la police a la réputation de tout savoir, il est impossible que le gouvernement ne sache pas qui est partie prenante à ce commerce. Mais arrêter des trafiquants serait dérouler un fil dont l'extrémité serait peut-être de trop hauts personnages. Tout le monde ferme donc les yeux.

En revanche, contrairement aux rumeurs répandues par les Etats-Unis, le Vietnam ne sert pas d'intermédiaire pour la drogue du Laos, sauf pour la marijuana. Cette politique relève de la prudence plus que de la morale car le trafic d'héroïne pourrait engendrer la corruption et le banditisme. Le seul fait significatif est que les Méo du Nord envoient maintenant leur opium à Canton, ce qui serait un moyen de pression de Hanoi sur Pékin.

\section{3.- Les Philippines}

Nouveau venu, les Philippines occupent une place de plus en plus importante, jouant presque un aussi grand rôle dans le transit que la Thaïlande. Seulement à la différence de la Thaïlande, le trafic ne se déroule pas sur un fond de lutte pour le pouvoir. Les participants désirent seulement s'enrichir dans un pays qui connaît la misère, et où les salaires des fonctionnaires sont ridiculement bas. C'est un discours du sénateur Ernesto Herrera, devant le Parlement, en septembre 1989, qui a mis le feu aux poudres. Il donna une liste d'une centaine de personnalités impliquées dans le trafic dans laquelle se trouvait, de manière surprenante, pratiquement tous les officiers de police et de l'armée chargés de la répression. Il affirmait, en outre, que $40 \%$ des membres de la police étaient impliqués dans le trafic. Toujours selon Ernesto Herrera les Philippines tendent à devenir une plaque tournante mondiale pour toutes les drogues expédiées aux Etats-Unis, Canada Australie et Nouvelle-Zélande. Le haschich provient du Pakistan et d'Afghanistan, le LSD de Taïwan, Hong Kong et Corée du Sud et 1héroïne ainsi que la marijuana de Thailande. Les Philippines se mettent elles-mêmes à la culture de la marijuana qui se pratiquerait déjà dans 45 de ses provinces. La Nouvelle Armée du peuple, branche armée du PC des Philippines, y participe aussi, trouvant là, comme le PC de Birmanie, un moyen facile de se financer. Cory Aquino, pour remercier les Etats- 
Unis d'être intervenus pour la protéger lors des différents coups d État, a tenté au maximum d'intensifier la lutte contre ces trafics. A la fin 1989, elle a nommé le général Alfredo Lim à la tête du National Bureau of avec l'ordre de traquer Investigation (équivalent au FBI américain) sans concession tous les trafiquants, quelle que soit leur fonction. Le NBI a obéi à la lettre mais se heurte à la police et aux forces armées. Cellesci s'opposent à son action, c'est-à-dire à l'arrestation des officiers compromis(9). Les accrochages meurtriers entre agents du NBI, la police et les militaires sont si fréquents qu'Herrera n'hésite pas à affirmer, en août. 1990, qu'il y a totale interpénétration entre trafiquants et forces de l'ordre. Le pays pourrait bientôt se transformer en une sorte de Colombie, les groupes de la drogue disposant d'armes lourdes, d'hélicoptères et possédant finalement un pouvoir politique, guerrier et financier suffisant pour contrer le gouvernement. C'est là sans doute un des cas les plus préoccupants et les plus significatifs que la bonne volonté ne suffit pas face à la montée de la pauvreté et à l'implication dans le trafic des forces de sécurité, donnée constante de la région.

\section{4.- L'extension en Chine : un changement d'échelle}

Après la désintégration $\mathrm{du} \mathrm{PCB}$, la frontière sino-birmane s'est transformée en un véritable caravansérail. Le commerce, sous forme de troc entre la Chine et la Birmanie, a provoqué un début de corruption du côté chinois. Cette pénétration dans la province du Yunnan, peuplée de montagnards qui n'ont jamais été complètement dociles envers Pékin, a permis l'établissement de réseaux de trafiquants. La filière part du Yunnan, va à Canton et se termine à HongKong ou Taïwan.

La Chine qui avait déjà saisi $300 \mathrm{~kg}$ d'héroïne, s'en est inquiétée dès la fin de 1989, soit quelques mois seulement après l'éclatement du PCB. Elle a demandé l'aide des EtatsUnis, ce qui visait aussi à prouver sa bonne foi lorsqu'elle affirme être elle-même une victime et non une participante au trafic. En novembre 1990, face à l'aggravation du problème, la Chine dit avoir saisi $1600 \mathrm{~kg}$ d'héroïne et décide que le seul moyen de lutte est la peine de mort. En juin 1991, il y a 40 exécutions, dont 27 dans le Yunnan et les autres à Canton. En août suivant, de l'opium a été saisi pour la première fois à Pékin. La toxicomanie et le gangstérisme, qui vont de pair avec le commerce, se développent néanmoins à pas de géant. La Chine, gui avait favorisé la culture du pavot en Birmanie voit ce plan se retourner contre elle. Les Chinois d'outre-mer trafiquants ne voient leur mère-patrie que comme un marché fort intéressant à une époque où l'Occident commence à être saturé et où le Triangle d'Or est durement concurrencé par le Croissant d'Or. Bref, l'avenir du trafic de drogue dans la région semble prospère et guère menacé par les politiques de lutte suivies par les différents pays. En effet, rien n'est fait pour mettre fin au trafic de la drogue.

Les Etats-Unis, principaux intéressés, ignorent délibérément le problème, se refusant à proposer des mesures concrètes et se contentant de plates dénonciations via la DEA. La Birmanie quant à elle est satisfaite : les différents groupes de trafiquants se battent entre eux et ne dirigent plus leurs actions contre le gouvernement. Quant à la Thaillande, elle argue du fait qu'elle ne peut pas plus empêcher le passage de la drogue, que les pays occidentaux ne peuvent en empêcher l'entrée. La Malaisie expose sa "répression", démontrée par les multiples exécutions par pendaison et marque ainsi son implication dans l'économie de transit. Il en va de même pour les Philippines, plongées dans l'anarchie. Le Laos, lui, nie purement et simplement les faits. Il n'y a donc que la Chine pour agir vigoureusement. L'héroïne d'Asie du Sud-Est dérange donc, car on ne peut nier le trafic massif, mais ni le gouvernement des Etats-Unis, ni ceux d'Europe ne veulent intervenir de manière ferme car l'Asie, qu'elle soit communiste ou 
fasse partie du "monde libre", à la différence de l'Amérique latine, est un client commercial et un enjeu géopolitique trop important.

\section{RÉSUMÉS}

Analysant la diversité des réseaux de trafiquants en Asie du Sud-Est, l'auteur montre que le trafic de la drogue est le fait de guérillas ethniques ou de groupes de bandits aux pseudo légitimations politiques en Birmanie alors qu'en Malaisie, à Singapour, aux Philippines et en Thaillande le trafic est de fait toléré, si ce n'est encouragé, par les gouvernements. Légitimée par les divers acteurs, la drogue est maintenant banalisée : elle rapporte avant tout des devises. Organisation du trafic par les militaires, ou/et les hommes politiques, arrangements en tout genre, faux-semblants de la répression à usage international caractérisent la situation de nombreux pays de la région. Les USA qui défendent leurs intérêts commerciaux ou stratégiques dans la région se contentent de plates dénonciations via la DEA alors que la CIA a encouragé ou encourage des groupes de narcotrafiquants. Seule la Chine, qui craint un retour de l'opiomanie, s'inquiète vraiment de cette situation, mais elle est déjà traversée par les différentes routes de la drogue.

Analysing the networks of traffickers in south-east Asia, the author shows how drug-traffic is the job of ethnic guerrilla or gangster groups pretending to political legitimacy in Burma while in Malaysia, in Singapore, in Philippines and in Thailand, traffic is tolerated de facto, if not encouraged, by these governments. Legitimated by different actors, drug is now banalised : above all, it brings in hard currencies. Traffic organisation by service-men and/or politicians, agreements of all kind, fake repression for international use, all characterise the situation of many of the area's countries. The United States, defending their commercial or strategic interests in the area, content themselves with denunciations via the DEA even though the CIA has encouraged or encourages at present narco-trafficking groups. China, fearing the return of opium addiction, is the only country to be really worried by the situation. However, it is already crossed by numerous drug-routes.

\section{INDEX}

Index géographique : Asie du Sud-Est

Mots-clés : contrebande et trafic, économie politique, mafias et crime organisé, drogue

\section{AUTEUR}

\section{MARTIAL DASSÉ}

Docteur d'Etat en science politique. Correspondant de La Croix en Asie du Sud-est, collaborateur de la revue Défense Nationale et à Etudes. Membre associé à La Fondation pour les Etudes de Défense Nationale. 